\title{
Cultural and Clinical Factors Associated with Antihypertensive Medication Adherence in Vietnamese Policemen
}

Asian/Pacific Island Nursing Journal

Volume 2(1): 11-18

(C)Author(s) 2017

http://digitalscholarship.unlv.edu/apin/

\author{
Wen-Wen Li ${ }^{a}$, Duong Thi Kim Nhungb, Wei-Shu Laic, and Shiow-Li Hwangd
}

\begin{abstract}
The purpose of this study is to characterize Vietnamese policemen with hypertension, to report the proportion of hypertension control and medication adherence in Vietnamese policemen, and to explore cultural and clinical factors related to antihypertensive medication adherence. A cross-sectional study design was used. A convenience sample of 100 policemen with hypertension was recruited in Vietnam. A total score of $80 \%$ or more on the Medication Adherence scale was defined as medication adherence. Medication adherence was reported in $64 \%$ of the participants, but hypertension control was found in only 53\%. Medication adherence was higher in the participants with higher perceived susceptibility to hypertension-related complications, higher perceived benefit of Western antihypertensive medications, longer duration of time since diagnosis, and fewer prescribed blood pressure lowering medications. Vietnamese policemen are at high risk for poor medication adherence and hypertension management. Culturally and clinically appropriate nursing interventions for hypertension management in Vietnamese policemen are needed.
\end{abstract}

Keywords: hypertension, medication adherence, Vietnamese, cultural factors

Hypertension (HTN) is an important public health challenge that affects approximately one billion people worldwide (World Health Organization [WHO], 2013). Kearney et al. (2005) analyzed global data and predicted that the number of adults with HTN would increase by about $60 \%$ to a total of 1.56 billion by 2025 . This indicates that prevention, treatment, and control of HTN should be a top health care priority.

The prevalence of HTN increases as an individual grows older. High cholesterol, tobacco use, increased alcohol and salt intake, living in an urban environment, becoming less physically active; and issues of poor stress management, diabetes, and obesity are all risk factors for developing HTN (Do, Geleijnse, Le, Kok, \& Feskens, 2015; WHO, 2013). The WHO (2013) reported that HTN is the most important modifiable risk factor for coronary heart disease, stroke, congestive heart failure, and end-stage renal disease. Each year, at least 9.4 million people die as a consequence of HTN worldwide (WHO, 2013). In addition to effective pharmacological treatments, optimal medication adherence and HTN control may reduce the incidence of HTN related complications (WHO, 2013).

Uncontrolled HTN can lead to complications, such as stroke, and increased health expenditures (Joint National Committee, 2003; WHO, 2013). Based on these estimates, 23 million cardiovascular deaths associated with uncontrolled HTN are projected by 2030 , with $85 \%$ occurring in developing countries (Mathers \& Loncar, 2006). A recent national survey in Vietnam estimated that the prevalence of HTN was 25-30\% for the general Vietnamese population (Do et al., 2015; Ha, Duy, Le, Khanal, \& Moorin, 2014; Son et al., 2012), making HTN a major public health problem in that country. Given this, helping hypertensive patients in developing coun-

\footnotetext{
${ }^{a}$ San Francisco State University, USA

b3/40 Hospital, Ho Chi Minh, Vietnam

${ }^{c}$ National Cheng Kung University, Taiwan

${ }^{d}$ Asia University, Taiwan

Corresponding Author:

Shiow-Li Hwang, RN, DNSc

Chair and Professor, Department of Nursing

Asia University

No. 500, Lioufeng Rd., Wufeng Dist.,

Taichung City 41354, Taiwan

Email: slhwang2@gmail.com
} 
tries, such as Vietnam, achieve better HTN control, particularly via optimal medication adherence, is important to prevent HTN-associated complications (Kearney et al., 2005; WHO 2013).

Compared to the general Vietnamese population, medication non-adherence may be more problematic in Vietnamese policemen because they have a different work culture, in particular, a stressful job and having to work day, evening, and night shifts (Violanti, 2015). A literature review failed to identify any studies that investigated the prevalence of HTN or examined clinical and cultural factors associated with antihypertensive medication adherence in Vietnamese policemen. Thus, this study was undertaken to learn more, with a goal of developing populationspecific interventions for successful HTN management.

\section{Background}

There are pharmacological and nonpharmacological treatments (e.g., lifestyle modification) for HTN management. Patients diagnosed with HTN usually need medication, in addition to lifestyle modification, to manage their blood pressure. When HTN is controlled, there is a decreased risk for further complications, such as stroke (WHO, 2013). Research has shown that for the general Vietnamese population who take antihypertensive medications, nearly half $(49 \%)$ did not adhere to their medication regimen (Ha et al., 2014). Thus, medication adherence remains an issue.

As previously mentioned, Vietnamese policemen may encounter more challenges to manage their HTN than Vietnamese patients in general because of the stress of their job (Violanti, 2015). Yet, a limited number of studies have been conducted to investigate the issues related to HTN management in this population. Given this, this study was implemented to investigate clinical and cultural factors associated with medication non-adherence to help advance the current nursing knowledge in taking care of the Vietnamese policemen. A significant contribution of this study was the use of cultural scales which were developed to study Asian immigrants who share similar cultural backgrounds, such as use of traditional Vietnamese herbs to treat disease, over-report of well-being to please health care providers, and emphasis on the impact of disease on family life (versus impact on an individual life which is more emphasized by Western culture). Therefore, the scales used in this study were culturally specific.

\section{Aims}

The purposes of this study were to characterize Vietnamese policemen with HTN, to report the proportion of HTN control and medication adherence, and to explore cultural and clinical factors related to antihypertensive medication adherence. Understanding this population by knowing their demographic and cultural backgrounds and identifying cultural factors for medication non-adherence are important in assisting nurses to adjust their interventions to be effective. In this paper, cultural factors refer to health perceptions of HTN and its treatment and social support, which will be discussed more thoroughly in the measurement section. Clinical factors included the duration of the HTN diagnosis, numbers of antihypertensive medications, measurement of blood pressures, and medication adherence. Medication adherence is defined in the literature as taking prescribed medications for treating HTN at a level of $80 \%$ or above (Ingersoll \& Cohen, 2008).

\section{Theoretical Framework}

The Health Belief Model (Figure 1) was modified to help predict medication adherence in Chinese and other Asian immigrants who share a similar cultural background with Chinese, such as Vietnamese (Li, Stewart, Stotts, \& Froelicher, 2005). Basically, the framework was derived from the following sources: (a) the Health Belief Model (Becker, 1974), (b) a literature review, (c) a series of qualitative pilot studies, and (d) the first author's years of clinical practice with Asian immigrants with HTN. The Health Belief Model is one of the first theories of health behavior and is commonly applied in the field of nursing (Becker, 1974). The theory was used to predict health behaviors, such as adherence to medications (Becker, 1974). The resources of the major concepts in our theoretical framework are the following. First, the concepts depicting susceptibility in general, susceptibility to specific diseases, and benefits of prescribed Western medications were derived from the Health Belief Model. Social support, in general, was derived from the literature review. Healthrelated social support and benefits of Vietnamese herbs were generated from the first author's qualitative pilot study (Li et al., 2005) and her clinical practice with Asian immigrants with HTN.

\section{Method}

This study was conducted in 2014 using a cross-sectional design. Two approaches were used: 1) self-report questionnaires to collect demographic information, cultural factors, and medication adherence; and 2) measures of blood pressure. Institutional review board approval was obtained from the Hospital Committee (the recruitment site) on Human Research. 


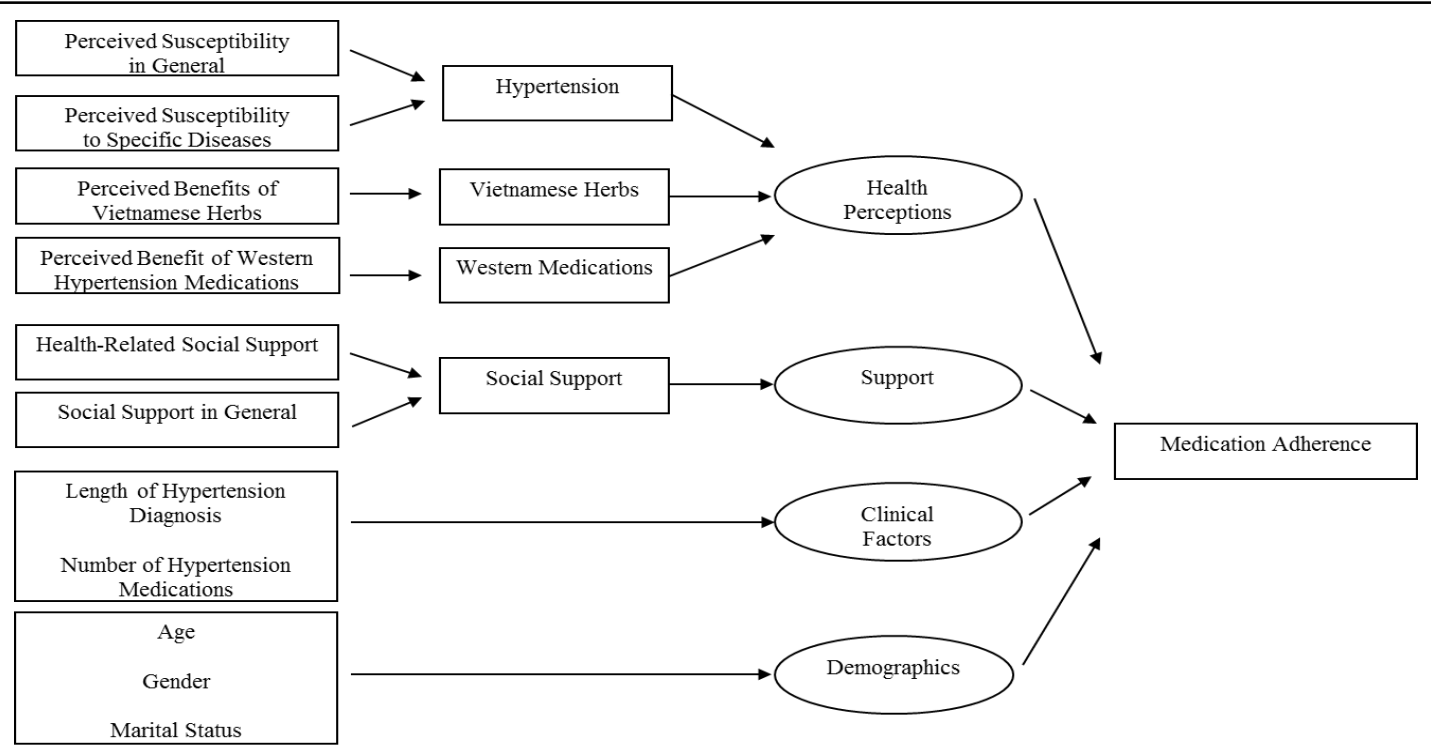

Figure 1. Theoretical Framework for Medication Adherence

\section{Setting}

A tertiary hospital under the General Logistics - Engineering Department, Ministry of Public Security in a metropolitan area was the recruitment site. The hospital is dedicated to providing health care primarily for policemen. Approximately 20 patients visit the hospital to check and monitor their blood pressure on a daily basis. However, according to the administrative team, the hospital does not have an effective program to help policemen manage their HTN as they do not have a good understanding of their patients' perceptions of HTN management and the competing priorities between the patients' jobs and their health needs.

\section{Sample}

A convenience sample of 100 Vietnamese policemen with HTN was recruited. Inclusion criteria were (a) being a Vietnamese policeman aged 18 years and older, (b) having a diagnosis of HTN, (c) having taken HTN medications for more than one month before study enrollment, and (d) being able to speak and read Vietnamese. Exclusion criteria were (a) being medically unstable (e.g., acute renal failure) or (b) having concurrent psychiatric problems (e.g., schizophrenia).

\section{Sample Size}

Using nQuery Advisor (Elashoff, 2007), when the sample size is 100 , the logistic regression test of $\alpha=.05$ (two-sided) will have more than $80 \%$ power to detect an odds ratio of 2.5. This assumes that one normally distributed covariate is being added to the model after adjustment for prior covariates, that the multiple correlations with the covariate already in the model is 0.2 , and that the proportion of success at the mean of that covariate is 0.2 .

\section{Measurements}

All questionnaires were translated by bicultural and bilingual experts. A translator took Chinese versions of questionnaires and translated them in Vietnamese. Once the Vietnamese version was ready, the second translator worked independently to translate the Vietnamese version back to Chinese. The two Chinese versions were then compared for inconsistencies by the authors. If there were inconsistencies, the authors worked together to compare the items and rewrote the items for a new edition. This procedure was repeated until the authors agreed on the revised items.

A total of six Likert scales were completed by the study participants to measure cultural factors, including perceived susceptibility to specific diseases, perceived susceptibility in general, perceived benefits of Vietnamese herbs, perceived benefits of western medications for HTN, health-related social support and social support in general. Detailed information about psychometric properties is included in Table 1. The validity of the questionnaires was reported previously (Li et al., 2005).

In addition to the six Likert scales, additional variables, including demographics, medication adherence, and duration of HTN diagnosis were included in the questionnaire. Demographics included age, gender, marital status, working status, educational 
Asian/Pacific Island Nursing Journal, Vol. 2, Iss. 1 [2017], Art. 2

Table 1. Psychometric Properties for Scale for Cultural Factors in Vietnamese Policemen

\begin{tabular}{lcccccc}
\hline Scale & $\begin{array}{c}\text { Perceived } \\
\text { Susceptibility } \\
\text { to Specific } \\
\text { Diseases }\end{array}$ & $\begin{array}{c}\text { Perceived } \\
\text { Susceptibility } \\
\text { in General }\end{array}$ & $\begin{array}{c}\text { Perceived } \\
\text { Benefits of } \\
\text { Vietnamese } \\
\text { Herbs }\end{array}$ & $\begin{array}{c}\text { Perceived Benefits of } \\
\text { Western Medications } \\
\text { for HTN }\end{array}$ & $\begin{array}{c}\text { Health- } \\
\text { Related } \\
\text { Social } \\
\text { Support }\end{array}$ & $\begin{array}{c}\text { Social } \\
\text { Support in } \\
\text { General }\end{array}$ \\
\hline No. of item & 5 & 2 & 4 & 2 & 7 & 6 \\
Mean $(S D)$ & $11.28(4.07)$ & $7.18(1.74)$ & $13.10(2.63)$ & $7.26(1.51)$ & $25.64(3.61)$ & $19.71(3.45)$ \\
Possible range & $5-25$ & $2-10$ & $4-20$ & $2-10$ & $7-35$ & $6-30$ \\
Cronbach's $\alpha$ & 0.83 & 0.74 & 0.57 & 0.90 & 0.75 & 0.70 \\
\hline
\end{tabular}

level, and income. Besides the questionnaire, participants' medical charts were reviewed for clinical factors, including number of HTN medications and HTN-related complications.

Six cultural factors were chosen to test their association with medication adherence; the factors were based on prior research (Li et al., 2005). These were measured by self-report.

Perceived Susceptibility to Specific Diseases was defined as the perception of developing complications if HTN is not controlled. The complications included heart attack, stroke, kidney disease, and cancer. A 5-item Likert scale was used with responses ranging from "strongly disagree" to "strongly agree." Scores were summed with higher scores indicating higher perceived susceptibility.

Perceived Susceptibility in General was defined as an understanding of the overall impact on daily life for the individual and family as a result of complications from uncontrolled HTN. This scale contained three items, with a 5-point score ranging from "strongly disagree" to "strongly agree." Scores were summed with higher scores reflecting higher perceived susceptibility.

Perceived Benefits of Vietnamese Herbs referred to the perception of the advantages of using Vietnamese herbs to prevent or treat their disease. This scale contained four items, with a 5-point score ranging from "strongly disagree" to "strongly agree." Scores were summed with higher scores reflecting higher benefits.

Perceived Benefits of Western Medications for HTN was defined as the perception of advantages of using Western HTN medications. This scale contained two items, with a 5-point score ranging from "strongly disagree" to "strongly agree." Scores were summed with higher scores reflecting higher benefits.

Health-Related Social Support was defined as the perception of health care support, such as receiving a ride to the clinic by family or friends. This scale contained seven items, scored on a 5-point scale, with responses ranging from "strongly disagree" to "strongly agree." Scores were summed with higher scores indicating more health-related social support.

Social Support in General referred to an individual perception of the general support of family members or friends. This contained six items, scored on a 5-point scale, with responses ranging from "strongly disagree" to "strongly agree." Scores were summed with higher scores indicating more social support.

\section{Measurement of Clinical Factors}

Duration of HTN Diagnosis (years) referred to the duration of time from the first diagnosis of HTN to the study interview. Participants provided this information.

Numbers of Antihypertensive Medications was defined as the medications that the patient took regularly for more than a month. It was recorded from chart review.

\section{Measurement of Medication Adherence}

The Medication Adherence Scale was used (Li et al., 2005). This scale contains three items regarding whether patients forgot, missed, or were careful about taking their medication. The scale was modified from Morisky, Green, and Levine (1986). The format of the questions and response choices were revised (e.g., instead of yes/no, a Likert scale ranging from "None of the time" to "All of the time" to better reflect Asian culture and Asian preferences in answering questions; $\mathrm{Li}$ et al., 2005).

\section{Measurement of Blood Pressure}

An Omron brand digital blood pressure mechanic (code: HEM-7201) was used to measure patient blood pressure following the standard processes by the Joint National Committee VII (Joint National Committee, 2003). Blood pressures were obtained twice. The values for systolic and diastolic blood pressures were averaged.

\section{Recruitment Plans and Procedures}

There were two approaches for participant recruitment. First, a flyer was posted in the hospital. It provided a contact phone number for potential participants to ask questions about the study and discuss their participation in the study. Second, the participants were invited by the health care provider to participate in the study. For either method, the potential 
Table 2. Demographic Profile of the Sample

\begin{tabular}{|c|c|c|c|}
\hline Variable & $\begin{array}{c}\text { Male } \\
(n=82) \\
n(\%)\end{array}$ & $\begin{array}{c}\text { Female } \\
(n=18) \\
n(\%)\end{array}$ & $\begin{array}{c}\text { Total } \\
(n=100) \\
n(\%)\end{array}$ \\
\hline \multicolumn{4}{|l|}{ Age $(M=50.88 ; S D=6.31)$} \\
\hline $18-30$ years & $3(3.36)$ & $0(0)$ & $3(3)$ \\
\hline $31-45$ years & $7(8.54)$ & $1(5.56)$ & $8(8)$ \\
\hline $46-60$ years & $72(87.80)$ & $17(94.44)$ & $89(89)$ \\
\hline \multicolumn{4}{|l|}{ Marital status } \\
\hline Married & $74(90.24)$ & $17(94.44)$ & $91(91)$ \\
\hline Divorce/Widow & $5(6.10)$ & $1(5.56)$ & $6(6)$ \\
\hline Single & $3(3.66)$ & $0(0)$ & $3(3)$ \\
\hline \multicolumn{4}{|l|}{ Living with family or friend } \\
\hline Yes & $78(95.12)$ & $17(94.44)$ & $95(95)$ \\
\hline No & $4(4.48)$ & $1(5.56)$ & $5(5)$ \\
\hline \multicolumn{4}{|l|}{ Occupation } \\
\hline Working office hours & $51(62.20)$ & $13(72.22)$ & $64(64)$ \\
\hline Working outside office hours & $31(37.80)$ & $5(27.78)$ & $36(36)$ \\
\hline \multicolumn{4}{|l|}{ Education level } \\
\hline Higher education & $15(18.29)$ & $4(22.22)$ & 19(19) \\
\hline Tertiary education & $67(81.71)$ & $14(77.78)$ & $81(81)$ \\
\hline \multicolumn{4}{|c|}{ Income/years (VND) $(M=115.30 ; S D=30.63)$} \\
\hline $50,000,000-75,000,000$ & $12(14.63)$ & $2(11.11)$ & $14(14)$ \\
\hline $76,000,000-100,000,000$ & $20(24.39)$ & $3(16.67)$ & $23(23)$ \\
\hline$>100,000,000 \mathrm{VND}$ & $50(60.98)$ & $13(72.22)$ & $63(63)$ \\
\hline \multicolumn{4}{|c|}{ The number of the people were supported by above income } \\
\hline $1-2$ & $12(14.63)$ & $3(16.67)$ & $15(15)$ \\
\hline $3-4$ & $60(73.17)$ & $11(61.11)$ & $71(71)$ \\
\hline $5-6$ & $10(12.20)$ & $4(22.22)$ & $14(14)$ \\
\hline
\end{tabular}

Note. $\mathrm{VND}=$ Vietnamese Dong

participants were screened by the research assistant for their eligibility. For those eligible, they were invited for an interview. On the day of the interview, the study purpose and voluntary nature of the study were explained to study participants. Afterword, their written consent was obtained. The participants were asked to rest for at least 5 minutes and then their blood pressure was checked twice. After their blood pressures were taken, they were asked to fill out the questionnaires. After the interview visit was completed, the research assistant reviewed and abstracted participants' medical records.

\section{Data Analysis}

All data were analyzed using IBM SPSS Statistics version 21 (IBM, Inc., Armonk, NY, USA). To describe the demographic and clinical variables, descriptive statistics were used. A multivariate logistic regression was used to examine the independent relationships of the 11 cultural and clinical factors with medication adherence. The results are presented as an odds ratio (OR) with $95 \%$ confidence interval (CI; Afifi \& Clark, 1997). In terms of an outcome, a total score $80 \%$ or more on the medication adherence scale was defined as adherence. Statistical significance was set at .05.
Descriptive statistics were used to screen data for missing values and outliers. Inconsistent data were checked and discrepancies verified. A multivariate logistic regression was used to examine the independent associations of eleven cultural, clinical, and demographic variables with medication adherence with results presented as $O R$ with $95 \%$ CI (Afifi \& Clark, 1997). Logistic regression analysis was chosen because of its not requiring an assumption of normality of data and ease of interpretation of study results in the form of $O R$ with 95\% CI (Afifi \& Clark, 1997).

\section{Results}

\section{Sample Characteristics}

Table 2 presents the demographic data. In general, the participants were young (mean age 50.88 \pm 6.31 ) with the majority being male $(82 \%)$, married $(91 \%)$, currently living with family or friends $(95 \%)$, and at least a high school education (81\%). Most considered themselves as healthy, but nearly twothirds did not have their blood pressure controlled (64\%). Participants had numbers of comorbidities including diabetes $(38 \%)$, liver disease $(23 \%)$ heart 
Table 3. Percent of Comorbidities

\begin{tabular}{lll}
\hline Other medical diagnoses $(n=100)$ & $n$ & $\%$ \\
\hline Stroke & 6 & 6 \\
Heart disease & 22 & 22 \\
Diabetes & 38 & 38 \\
Chronic obstructive pulmonary disease & 3 & 3 \\
Peripheral vascular disease & 2 & 2 \\
Kidney disease & 14 & 14 \\
Liver disease & 23 & 23 \\
Cancer & 1 & 1 \\
\hline
\end{tabular}

Table 4. Multivariate Logistic Regression of Cultural and Clinical Factors for Medication Adherence

\begin{tabular}{|c|c|c|c|}
\hline Variables & $P$ & $O R$ & $\begin{array}{c}95 \% \mathrm{CI} \\
\text { (lower, upper) }\end{array}$ \\
\hline Higher perceived susceptibility to specific disease & $<0.001$ & 1.41 & $1.17,1.69$ \\
\hline Higher perceived susceptibility in general & 0.869 & 1.03 & $0.73,1.46$ \\
\hline Lower perceived benefit of Vietnamese herbs & 0.577 & 1.07 & $0.85,1.34$ \\
\hline Higher perceived benefit of western medications for hypertension & 0.002 & 1.91 & $1.26,2.90$ \\
\hline Higher perceived health-related social support & 0.860 & 1.01 & $0.87,1.19$ \\
\hline Higher perceived social support in general & 0.122 & 1.15 & $0.96,1.39$ \\
\hline Older age & 0.187 & 0.94 & $0.85,1.03$ \\
\hline Longer duration of hypertension diagnosis & 0.016 & 1.30 & $1.05,1.62$ \\
\hline Lower number of prescribed hypertension medications & 0.014 & 3.62 & $1.29,10.14$ \\
\hline Female & 0.250 & 0.34 & $0.05,2.14$ \\
\hline Married & 0.295 & 0.46 & $0.11,1.97$ \\
\hline
\end{tabular}

disease $(22 \%)$, and kidney disease (14\%). The data indicated that participants were at high risk for severe complications, such as stroke (see Table 3).

The majority (63\%) earned 100 million Vietnamese Dong (approximately \$4,458 USD) annually. Roughly three-quarters $(71 \%)$ reported that they needed to use their single income to support 3-4 people. Nearly twenty participants (17\%) used Vietnamese herbs to treat their HTN.

The mean duration of HTN diagnosis was 5.15 years $(S D, 4.4)$ ranging from 1-29 years. The mean number of prescribed HTN medications was 0.56 ( $S D, 0.67)$, ranging from 0-2. Proportions of medication adherence and blood pressure control were $64 \%$ and $53 \%$ respectively.

\section{Cultural Factors and Non-Medication Adherence}

A multiple regression analysis was used to evaluate the independent effects of 11 variables for their association with medication adherence, and four variables were statistically significant: higher perceived susceptibility to specific disease $(O R=1.41$ [95\% CI, 1.17-1.69]), higher perceived benefit of western HTN medications $(O R=1.91$ [95\% CI, 1.26$2.90])$, a longer duration of HTN diagnosis $(O R=$
1.30 [95\% CI, 1.05-1.62]), and a lower number of prescribed HTN medications taken on a regular basis $(O R=3.62$ [95\% CI, 1.29-10.14]; Table 4).

\section{Discussion}

Due to a limited number of studies that have been conducted with Vietnamese exploring cultural and clinical factors associated with antihypertensive medication adherence, we were unable to compare our study findings in Vietnamese policemen with the general Vietnamese population. Instead, we compared these findings with other populations who share similar cultural backgrounds, such as Taiwanese and Chinese immigrants in the United States. It is suggested that future studies be done in the general Vietnamese population to explore potential issues related to HTN management.

In terms of medication adherence, only $64 \%$ of the participants were adherent, which is much lower than $80 \%$ found in the general U.S. population. This is concerning because research has shown that $80 \%$ of medication adherence is necessary for good HTN control (Ingersoll \& Cohen, 2008). The proportion of HTN control in this population was also low (53\%), compared to $70 \%$ of the U.S. population. Therefore, it is crucial to develop a culturally and 
clinically appropriate HTN management program for Vietnamese policemen to achieve better medication adherence and optimal blood pressure control.

In general, participants had low perceived susceptibility to specific diseases (e.g., a mean score of 11.28 out of a maximal score of 25). This may be due to this population's over-estimation of their wellness (i.e., being healthier than the general population, but in reality, they are not) and inability to have more time to pay attention to health care because of a stressful job and working multiple shifts (Violanti, 2015). In addition, the asymptomatic nature of HTN may be an important contributor to their suboptimal HTN management. This warrants future studies to discover solutions to overcome the aforementioned barriers, including low perceived susceptibility to complications and asymptomatic HTN, to help improve awareness of HTN-related complications, which in turn improves HTN management.

Medication adherence was higher in the participants who had higher perceived susceptibility to specific disease. This result was similar to the study findings conducted in a Taiwanese population ( $\mathrm{Li}$, Kuo, Hwang, \& Hsu, 2012) and a Chinese immigrant population in the United States ( $\mathrm{Li}$ et al., 2006). The findings indicated that HTN management education should be implemented in the Vietnamese policemen population to increase their perception about the severity of complications secondary to non-adherence to HTN medications and uncontrolled HTN.

Adherence was higher in the participants who had higher perceived benefits of Western HTN medications. This result was similar to studies conducted in Taiwanese (Li et al., 2012) and Chinese immigrants in the United States (Li, Stewart, Stotts, \& Froelicher, 2006). Given this, offering medicationrelevant health education, such as the emphasis on efficiency and benefits of Western HTN medications in lowering blood pressure, should be offered to increase participants' knowledge about Western medications, which in turn improves their medication adherence and HTN management.

To increase the policemen's awareness of the severity of HTN-related complications and their perceived benefits of Western antihypertensive medications, culturally and clinically appropriate nursing interventions for HTN management, such as offering on-site health education classes, is needed to encourage participation. Ideally, on-site classes can be offered in a conference room adjacent to the police department. Other on-site classes, such as stressreduction exercise programs, can also be offered to alleviate their stress-aggravated HTN.

The duration of HTN diagnosis ranged widely from 1-29 years which adds complexities for nurses working with this population to improve their health literacy and self-management of HTN. The prediction model showed that with a shorter duration of a HTN diagnosis, medication adherence was poorer. This may also indicate that those who were recently diagnosed had a much lower level of knowledge about HTN management than those who had been living with HTN for many years. Given this, we recommend that nurses spend significant time in educating newly diagnosed patients about the importance of medication adherence and HTN management. An alternative is to have peer-to-peer health education offered by policemen with a longer diagnosis to those with a new diagnosis, under the supervision of a nurse.

In terms of the number of HTN medications, the lower number predicted higher medication adherence. This was consistent with the findings in the Taiwanese study (Li et al., 2012) and the Chinese immigrant study (Li et al., 2006). It is recommended that a nurse should help monitor the number of HTN medications that patients take and work with primary care providers to reduce the number of prescribed medications. This is especially important for those who are busy with stressful jobs.

In summary, Vietnamese policemen have a specific work culture with regular exposure to stressors, coupled with their perception of better health than the general Vietnamese population. Findings showed that this population had a high prevalence of comorbidities; hence, their health was not as good as they perceived. Being stressed and overestimating their health put these policemen at high risk for poor medication adherence and HTN management. Thus, it is crucial to develop culturally and clinically appropriate interventions to help this population achieve optimal blood pressure control. Several factors should be taken into account, including improving an individual's perceived susceptibility to specific diseases, improving perceived benefits of HTN medications, and prescribing a lower number of HTN medications. Regarding the duration of HTN diagnosis, for patients with a short duration of a HTN diagnosis (e.g., within one year), a more intense health education program should be implemented to ensure the success of HTN management.

\section{Limitations and Recommendations}

A cultural scale, Perceived Benefits of Vietnamese Herbs, had relatively low Cronbach's $\alpha$ as 0.57 . This is considered as acceptable for the exploratory phase of the research project like this study (Nunnally \& Bernsteinduration, 1994). However, it is recommended that this scale be further tested in Vietnamese policemen across different geographic areas, such as urban and rural areas, to further test its reliability, which in turn establishes a more robust prediction model. 


\section{Conclusion}

Participants in this study had a high prevalence of comorbidities, such as diabetes, and poor HTN management. However, they reported betterperceived wellness than their general counterparts in Vietnam. This contraindication indicates that Vietnamese policemen were unaware of their poor HTN management. Besides their unrealistic health perceptions, the asymptomatic nature of HTN may be another contributor to their lack of motivation to better manage their HTN. Given this, establishing a prediction model for medication adherence is important for nurses to help identify those who are at risk for poor medication adherence and HTN management and address this with culturally and clinically appropriate interventions. In addition, onsite educational programs should be offered to help this population better control their HTN. Our prediction model warrants further tests in other communities and cities in $\mathrm{Vi}$ etnam, to establish a more robust program to help design culturally and clinically appropriate interventions for Vietnamese policemen. The same prediction model and intervention program may be applied to other populations who share similar cultural backgrounds, such as Taiwanese or Chinese policemen.

\section{Implications for Clinical Practice}

This study found that participants perceived themselves as healthier than they were, with less than optimal medication adherence to manage their HTN. Nurses need to work closely with these types of patients to design and implement effective HTN management programs which are culturally appropriate and can meet their health needs. In addition, educating patients about the importance of taking their HTN medications regularly is critical to ensure their effectiveness and to prevent potentially serious complications. The goal is to help Vietnamese policemen and other Asian and Pacific Islander policemen populations, who share similar cultural backgrounds, achieve optimal medication adherence and HTN control.

\section{Acknowledgment}

The authors wish to thank all Vietnamese policeman patients who participated in this study.

\section{References}

Afifi, A. A., \& Clark, V. (1997). Computer-aided multivariate analysis (3rd ed.). New York: Chapman \& Hall/CRC.

Becker, M. H. (1974). The Health Belief Model and sick role behavior. Health Education Monographs, 2, 409-419. doi:10.1177/109019817400200407

Do, H. T., Geleijnse, J. M., Le, M. B., Kok, F. J., \& Feskens, E. J. (2015). National prevalence and asso- ciated risk factors of hypertension and prehypertension among Vietnamese adults. American Journal of Hypertension, 28, 89-97. doi:10. 1093/ajh/hpu092

Elashoff, J. D. (2007). nQuery advisor version 7.0 user's guide. Los Angeles, CA.

Ha, N. T., Duy, H. T., Le, N. H., Khanal, V., \& Moorin, R. (2014). Quality of life among people living with hypertension in a rural Vietnam community. BMC Public Health, 14(833). doi:10.1186/1471-245814-833

Ingersoll, K. S., \& Cohen, J. (2008). The impact of medication regimen factors on adherence to chronic treatment: A review of literature. Journal of Behavioral Medicine, 31, 213-224. doi:10. 1007/s10865-007-9147-y

Joint National Committee. (2003). The seventh report of the Joint National Committee on prevention, detection, evaluation, and treatment of high blood pressure (NIH Publication No. 03-5233). Bethesda, MD: National Institutes of Health.

Kearney, P. M., Whelton, M., Reynolds, K., Muntner, P., Whelton, P. K., \& He, J. (2005). Global burden of hypertension: Analysis of worldwide data. Lancet, 365(9455), 217-223. doi:10.1016/S01406736(05)70151-3

Li, W. W., Kuo, C. T., Hwang, S. L., \& Hsu, H. T. (2012). Factors related to medication non-adherence for patients with hypertension in Taiwan. Journal of Clinical Nursing, 21, 1816-1824. doi:10.1111/ j.1365-2702.2012.04088.x

Li, W. W., Stewart, A. L., Stotts, N. A., \& Froelicher, E. S. (2005). Cultural factors and medication compliance in Chinese Immigrants who are taking antihypertensive medications: Instrument development. Journal of Nursing Measurement, 13, 231-252. doi:10.1891/jnum.13.3.231

Li, W. W., Stewart, A. L., Stotts, N., \& Froelicher, E. S. (2006). Cultural factors associated with antihypertensive medication adherence in Chinese immigrants. The Journal of Cardiovascular Nursing, 21, 354-362. doi:10. 1097/00005082-200609000-00005

Mathers, C. D., \& Loncar, D. (2006). Projections of global mortality and burden of disease from 2002 to 2030. PLoS Medicine, 3(11), e442.

Morisky, D. E., Green, L. W., \& Levine, D. M. (1986). Concurrent and predictive validity of a selfreported measure of medication adherence. Medical Care, 24, 67-74. doi:10.1097/00005650198601000-00007

Nunnally, J., \& Bernstein, I. (1994). Psychometric theory (3rd ed.). New York, NY: McGraw Hill.Son, P. T., Quang, N. N., Viet, N. L., Khai, P. G., Wall, S., Weinehall, L., ... Byass, P. (2012). Prevalence, awareness, treatment and control of hypertension in Vietnam-results from a national survey. Journal of Human Hypertension, 26, 268280. doi:10.1038/jhh.2011.18

Violanti, J. M. (2015). Police psychological trauma. Retrieved from http://www.stevedavis.org/ sollart12.html

World Health Organization. (2013). A global brief on hypertension. Retrieved from http://apps.who.int/ iris/bitstream/10665/79059/1/WHO_DCO_WHD 2013.2_eng.pdf?ua $=1$ 